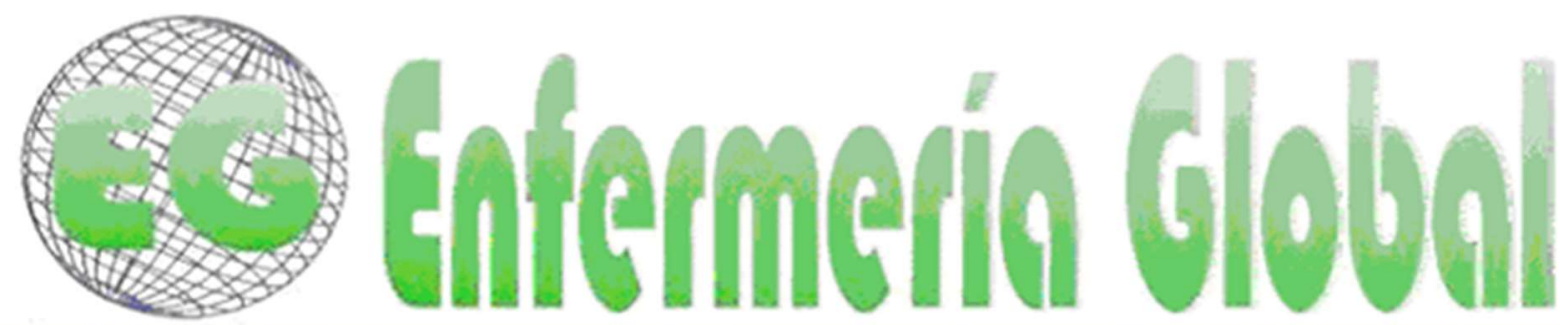

\title{
ORIGINALES
}

\section{El miedo al contagio de covid-19, como mediador entre la exposición a las noticias y la salud mental, en población peruana}

Fear of COVID-19 infection, as a mediator between exposure to news and mental health, in the Peruvian population

Henry Santa-Cruz-Espinoza ${ }^{1}$

Gina Chávez-Ventura ${ }^{1}$

Julio Domínguez-Vergara²

Elizabeth Dany Araujo-Robles ${ }^{3}$

Haydee Mercedes Aguilar-Armas ${ }^{4}$

Velia Vera-Calmet ${ }^{4}$

${ }^{1}$ Docente investigador adscrito al Instituto de Ciencia y Tecnología de la Universidad César Vallejo, Perú. hsantacruze@gmail.com

${ }^{2}$ Docente Investigador de la Universidad Tecnológica del Perú.

${ }^{3}$ Escuela de Posgrado, Universidad Peruana Cayetano Heredia, Perú.

${ }^{4}$ Docente de la Escuela de Psicología de la Universidad César Vallejo, Perú

\section{https://doi.org/10.6018/eglobal.489671}

Recibido: $17 / 08 / 2021$

Aceptado: 28/09/2021

\section{RESUMEN:}

Introducción: En esta pandemia por covid-19 se deben conocer las medidas de protección contra la enfermedad y las políticas impuestas por el gobierno; empero, los medios de comunicación también informan sobre las muertes y el desabastecimiento de los servicios de salud, pero se ignora su impacto en la salud mental de la población.

Objetivo: Determinar si el miedo al contagio de la covid-19 actúa como mediador entre la exposición a las noticias sobre la pandemia y la salud mental en población peruana.

Método: Estudio explicativo con variables observables, donde participaron 541 personas seleccionadas con un muestreo no probabilístico. Respondieron una ficha sociodemográfica y las escalas: Mental Health Inventory-5 (MHI-5) y Fear of Covid-19 Scale. Los datos fueron procesados mediante los programas IBM SPSS Statistics 25 y Macro PROCESS para SPSS; se utilizó la regresión lineal y bootstrapping de 10000 simulaciones.

Resultados: El número de horas viendo y/o escuchando información de la covid-19 es un buen predictor de la variable mediadora de miedo a la covid-19 $\left(\beta=, 75 ; t=3,77, p<, 001^{* *}\right)$; a su vez, esta tiene un efecto predictor sobre la salud mental $\left(\beta=-, 24 ; t=-13,57, p<, 001^{* *}\right)$; sin embargo, el número de horas de exposición a la información de la covid-19 no tuvo un efecto directo positivo en la salud mental $(\beta=-, 10 ; t=-1,184, p=, 23)$.

Conclusión: El miedo a la covid-19 tiene un efecto mediador total entre la exposición a las noticias sobre la pandemia y la salud mental en la población peruana.

Palabras clave: Pandemia, Coronavirus, Medios de comunicación, Salud psicológica. 


\begin{abstract}
:
Introduction: In this COVID-19 pandemic, protective measures against the disease and governmentimposed policies should be known. However, the media also report on deaths and health service shortages, but their impact on the mental health of the population is ignored.

Objective: To determine whether fear of COVID-19 infection acts as a mediator between exposure to news about the pandemic and mental health in the Peruvian population.

Method: Explanatory study with observable variables in which 541 persons selected by nonprobabilistic sampling participated. They responded to a sociodemographic file and the following scales: Mental Health Inventory-5 (MHI-5) and Fear of COVID-19 Scale. Data were processed using IBM SPSS Statistics 25 and Macro PROCESS for SPSS programs; linear regression and bootstrapping of 10000 simulations were used.

Results: The number of hours watching and/or listening to covid-19 information is a good predictor of the COVID-19 fear mediator variable $\left(\beta=, 75 ; \mathrm{t}=3.77, p<.001^{* *}\right)$. In turn, this has a predictor effect on mental health $\left(\beta=-, 24 ; t=-13.57, p<.001^{* *}\right)$. However, the number of hours of exposure to COVID-19 information had no direct positive effect on mental health $(\beta=-.10 ; t=-1.184, p=.23)$.

Conclusion: Fear of COVID-19 has a total mediating effect between exposure to pandemic news and mental health in the Peruvian population.
\end{abstract}

Keywords: Pandemic, Coronavirus, Media, Psychological health.

\title{
INTRODUCCIÓN
}

En diciembre del año 2019, se informó a la población mundial que un nuevo virus llamado SARS-COV-2 (Severe Acute Respiratory Syndrome Coronavirus), estaba dando origen a una nueva pandemia, originada en la región de Wuhan de China, catalogada como la enfermedad de Coronavirus, covid-19 (1). Tres meses después, la OMS informó que este virus se encontraba disperso en 159 países, causando hasta ese entonces más de 8000 muertes ${ }^{(1)}$. El principio agresivo y acelerado de esta enfermedad es considerado una emergencia internacional de salud pública, a consecuencia de la excesiva presencia de casos positivos y de muertes por contagio, situación que en la actualidad se mantiene ${ }^{(2)}$.

La aparición del SARS-CoV-2, su rápida propagación y mutaciones, han generado un fuerte impacto en el sistema de salud, en la economía, en el avance académico y en los estilos de vida en general ${ }^{(3)}$, por lo que distintos países tuvieron que adoptar como medida preventiva el aislamiento social, el cual generó consecuencias en la salud mental de su población (4).

Las diversas reacciones emocionales que presentó la población en general, durante la epidemia de enfermedad por covid-19, fueron: depresión, ansiedad, estrés ${ }^{(5)}$, miedo y otras reacciones de afectividad negativa ${ }^{(6)}$; además, se han recrudecido los síntomas de quienes padecían antecedentes psicológicos, agravando y agudizando su situación $^{(6)}$.

También, los pacientes infectados con covid-19 presentaron problemas psicopatológicos de ansiedad, depresión y estrés postraumático ${ }^{(7)}$. De manera similar, los profesionales de salud, que atienden en primera línea a pacientes con covid-19, vivenciaron miedo, ansiedad y depresión ${ }^{(8)}$, pero además experimentaron trastorno por estrés postraumático ${ }^{(9)}$, agotamiento, miedo a contagiar la enfermedad y aumento de dependencia a sustancias ${ }^{(9)}$.

Un importante predictor de la ansiedad y depresión es la intolerancia a la incertidumbre ${ }^{(10)}$; y las tres variables en conjunto con el estrés, median la relación 
entre el miedo a la covid-19 y la actitud optimista (1). Definitivamente, la ambigüedad influye en la emoción de miedo, sin embargo, disponer de información que ofrezca credibilidad favorece las conductas adaptativas, reduce la ansiedad (11) y permite tomar decisiones oportunas, minimizando el riesgo para el contagio (12). Así, la información recibida del personal de salud, contribuye a experimentar menos temor ${ }^{(13)}$ y una mejor salud mental (14).

No obstante, la principal fuente de información que el público general consume sobre la covid-19 son la televisión y las redes sociales ${ }^{(12)}$, que divulgan, ocasionalmente, información respecto a los nuevos descubrimientos de la enfermedad, pautas para la prevención $\mathrm{y}$, con mayor regularidad, las medidas gubernamentales para la contención del contagio; así como también información sobre los decesos, las cifras de contagiados, el desacato de las medidas de control, la precariedad de los servicios de salud y su desabastecimiento para la atención oportuna de los casos.

Paralelamente a ello, ha surgido en internet una sobreabundancia de información, que en algunas ocasiones es correcta, pero también se presenta la infundada, carente de sustento racional, o imprecisa denominada infodemia (15). Está marcada por la intencionalidad dudosa, y por la difusión de información no verificada que se extiende con suma facilidad, creando confusión y engaño, entre quienes no tienen competencias informacionales (16). Se divulga información asociada al miedo, especulación y rumores, transmitida a través de las nuevas tecnologías de la información y medios de comunicación, que pueden ocasionar pánico, rechazo a acatar las medidas gubernamentales para el control del contagio (17) y que las personas no tengan orientación fidedigna cuando la requieran ${ }^{(15)}$.

Los medios de comunicación son importantes impulsores en la propagación del miedo ante el SARS-CoV-2 ${ }^{(12)}$. Estos hallazgos confirmaron los de un estudio previo donde se obtuvo que la frecuencia de exposición a los estímulos presentados tanto en los medios de comunicación, como en las comunicaciones interpersonales, facilitó la memoria de los estímulos e instigó el miedo, en lugar de producir su atenuación, respecto al zika ${ }^{(18)}$. De igual modo, otra investigación experimental reportó que los medios de comunicación contribuyen a exacerbar los miedos en estudiantes universitarios ${ }^{(19)}$.

En síntesis, se dispone de algunas evidencias sobre el impacto que generan los medios de comunicación en el miedo, y se desconoce la afectación que éste puede tener en la salud mental, la cual es de suma importancia por ser un aspecto integral y esencial de la salud, referido al bienestar bio-psico-social y no únicamente a la ausencia de enfermedad ${ }^{(20)}$. En el contexto actual de la pandemia, de acuerdo a una revisión sistemática, 1 de cada 5 personas se encuentra en riesgo de desarrollar problemas psicológicos importantes ${ }^{(4)}$.

Por ello, en el escenario actual de incertidumbre, es necesaria la autoobservación de las propias emociones, entre ellas el miedo (21). Estudios reportan que el miedo al covid-19 está vinculado a la presentación de síntomas como la depresión y la ansiedad ${ }^{(22,23)}$, el insomnio ${ }^{(22)}$, el trastorno de la adicción al internet ${ }^{(23)}$, así como una relación negativa con la calidad de vida en embarazadas (24). Por ello, surge la necesidad de desarrollar un estudio orientado a determinar si el miedo al contagio de la covid-19 actúa como mediador entre la exposición a las noticias y la salud mental en la población peruana. 
En el presente estudio se plantean tres hipótesis: $\left(\mathrm{H}_{1}\right)$ el miedo a la covid-19 se relaciona directamente con la exposición a noticias sobre la covid-19 $(12,18,19)$ y negativamente con la salud mental $(22,23,24) ;\left(\mathrm{H}_{2}\right)$ la exposición a noticias sobre la covid-19 se relaciona negativamente con la salud mental ${ }^{(25)}$, y $\left(\mathrm{H}_{3}\right)$ el miedo a la covid-19 ejerce un efecto mediador entre la exposición a las noticias sobre la covid-19 y la salud mental.

Los hallazgos contribuirán a orientar la toma de decisiones, especialmente para favorecer grupos vulnerables, y al mismo tiempo servirán como base para realizar planificaciones estratégicas de intervenciones psicológicas y para la toma de conciencia de la responsabilidad social que debe ser inherente a la difusión de la información, tanto de los medios de comunicación, como en las redes e interacciones sociales.

\section{MÉTODO}

\section{Participantes y procedimiento}

Este es un estudio explicativo con variables observables desarrollado en una población general de seis departamentos del Perú (La Libertad, Lima, Lambayeque, Piura, Tumbes y Cajamarca). Se utilizó un muestreo no probabilístico por conveniencia y se consideraron los siguientes criterios de inclusión: 1) personas mayores de 18 años; 2) residencia en Perú y 3) aceptar voluntariamente participar en el estudio. La recopilación de información se realizó mediante datos de encuestas en línea mediante redes sociales desde el 15 de junio al 2 de octubre del 2020.

La muestra se constituyó por 541 personas, de quienes el 31,6\% fueron varones y $68,4 \%$ mujeres con una edad media de 30,13 años (DE $=10,31$ ). La mayoría de participantes fueron solteros $(n=354 ; 65,4 \%)$, seguidos de casados $(n=105 ; 19,4 \%)$, convivientes $(\mathrm{n}=64 ; 11,8 \%)$ y divorciados $(\mathrm{n}=18 ; 3.3 \%)$. La mayoría de los participantes no ha tenido diagnóstico de covid-19 $(n=530 ; 98 \%)$ y la mayoría de los participantes se informan mediante noticias de covid-19 de 1 a 3 horas diarias $(51,2 \%)$. En la tabla 1 se detallan las características sociodemográficas de la muestra.

Tabla 1. Datos sociodemográficos de la muestra

\begin{tabular}{lcc}
\hline Características & $\mathbf{n = 5 1 7}$ & $\mathbf{\%}$ \\
\hline Sexo & & \\
$\quad$ Masculino & 171 & 31,6 \\
Femenino & 370 & 68,4 \\
Estado civil & & \\
$\quad$ Soltero(a) & 354 & 65,4 \\
Casado(a) & 105 & 19,4 \\
Conviviente & 64 & 11,8 \\
Divorciado(a) & 18 & 3,3 \\
Exposición a las noticias sobre covid-19 & & \\
1 a 3 horas & 277 & 51,2 \\
3 a 5 horas & 114 & 21,1 \\
5 a 7 horas & 55 & 10,2
\end{tabular}


más de 7 horas

Contagio por covid-19

Sí

11

2

No

530

98

Diagnóstico de covid-19 en algún familiar

No

320

59,1

Tengo familiares lejanos contagiados por covid19

Tengo familiares cercanos contagiados por covid-19

Diagnóstico de covid-19 en amigos

No

$\mathrm{Si}$

Situación laboral

Trabajo fijo

Trabajo temporal

\section{Instrumentos y medición}

\section{Ficha sociodemográfica}

Esta ficha recoge información sociodemográfica de los participantes como sexo, edad, estado civil, contagio por covid-19, diagnóstico de covid-19 en algún familiar, diagnóstico de covid-19 en amigos y situación laboral. La exposición a las noticias sobre covid-19, se midió mediante la pregunta: "Durante las últimas semanas ¿cuántas horas has pensado, visto y/o escuchado información sobre el coronavirus?" bajo una escala de 4 puntos que va desde 1 ( 1 a 3 horas), 2 ( 3 a 5 horas), 3 ( 5 a 7 horas) y 4 (más de 7 horas).

\section{Mental Health Inventory-5 (MHI-5)}

El instrumento fue creado por Veit y Ware en 1983 para su uso en población general. La escala posee cinco ítems y utiliza una escala Likert de 4 puntos (nunca $=1$, algunas veces $=2$, muchas veces $=3$ y siempre $=4$ ), la puntuación varía de 5 a 20 . El instrumento evalúa la presencia de bienestar y angustia. Presenta una solución bidimensional con cargas factoriales superiores a 0,57 , una consistencia interna de alfa de 0,83 y es aplicable en población general adulta y adolescente (26).

Para este estudio, la estimación de la confiabilidad del instrumento MHI-5 se realizó mediante el coeficiente omega $(\omega=0,82$; IC- $95 \%-0,80-0,85)$, cuya evidencia de consistencia interna fue adecuada en las puntuaciones de la medida usada en el estudio.

\section{Fear of COVID-19 Scale}

La escala de miedo al covid-19 fue creada por Ahorsu, Chung-Ying, Imani, Saffari, Griffiths y Pakpour en el 2020, es un instrumento unidimensional de siete ítems. 
Evalúa temores de COVID-19 en población general y utiliza una escala Likert de 5 puntos $(1=$ muy en desacuerdo a $5=$ muy de acuerdo). La puntuación oscila entre 7 a 35 , donde elevados puntajes indican mayor miedo a la covid-19. Las propiedades psicométricas de la escala de miedo a la covid-19 en una muestra peruana fueron obtenidas por Huarcaya-Victoria, Villarreal-Zegarra, Podesta y Luna-Cuadros (27) quienes reportaron un modelo bifactor que consta de un factor general y dos factores específicos.

El primer factor se denominó reacciones de miedo emocional y el segundo, expresiones somáticas de miedo a la covid-19. El modelo presentó valores adecuados en varios índices de ajuste (CFI = 0,988; TLI = 0,964; RMSEA = 0,075 [0,054-0,098]). Para la confiabilidad de consistencia interna, se calculó el coeficiente omega, obteniéndose 0,94 para escala total y 0,91 y 0,89 para las escalas de reacciones de miedo y expresiones somáticas, respectivamente ${ }^{(44)}$.

En el presente estudio, se calculó la confiabilidad de la escala de miedo a la covid-19, mediante el coeficiente omega, ( $\omega=0,85$; IC- $95 \%-0,83-0,87$ ), lo que indica un buen nivel de consistencia interna.

\section{Análisis de datos}

El procesamiento de datos se hizo mediante el programa estadístico IBM SPSS Statistics 25 mediante el cual se calcularon la el valor mínimo, máximo, media (M) y la desviación típica (DE). El análisis mediacional se hizo mediante el programa Macro PROCESS para SPSS para probar los efectos de mediación del miedo a la covid-19 entre la exposición a las noticias sobre covid-19 y la salud mental. Para este análisis, se tomó en cuenta el cálculo del efecto indirecto del mediador. La significancia estadística de la variable mediadora se obtuvo mediante un bootstrapping de 10000 simulaciones $(p<0,05)$. Los intervalos de confianza (IC) al $95 \%$ que no se encontraban a ambos lados de cero, se consideraron estadísticamente significativos.

\section{Aspectos éticos}

Este estudio cuenta con la aprobación del Comité de Ética de la Universidad César Vallejo. Se solicitó el consentimiento informado de cada participante, quienes lo hacen de manera voluntaria y conocen del anonimato de la información brindada.

\section{RESULTADOS}

La Tabla 2 muestra las estadísticas descriptivas para la muestra total, así como las correlaciones entre las tres variables. Solo la correlación entre miedo a la covid-19 fue significativa y mostró un efecto grande. 
Tabla 2. Estadística descriptiva y correlaciones bivariadas de las variables de estudio

\begin{tabular}{|c|c|c|c|c|c|c|c|}
\hline $\mathbf{N}^{\circ}$ & Variables de estudio & Min & Máx & $M$ & $\mathrm{DE}$ & 2 & 3 \\
\hline 1 & Miedo a la covid-19 & 7 & 35 & 17,8 & 5,4 & $0.515^{* *}$ & 0,16 \\
\hline 2 & Salud mental & 5 & 19 & 14,9 & 2,6 & - & $-0,126$ \\
\hline 3 & $\begin{array}{c}\text { Exposición a las noticias } \\
\text { sobre covid- } 19\end{array}$ & 1 & 4 & 1,94 & 1,2 & & - \\
\hline
\end{tabular}

** Significativa al nivel de 0,01 (de dos colas); Min = Valor mínimo, Máx = Valor máximo, $\mathrm{M}=$ Media, DE = Desviación típica

Figura 1: Modelo de mediación del miedo a la covid-19 en la relación de la exposición a las noticias sobre covid- 19 y la salud mental.

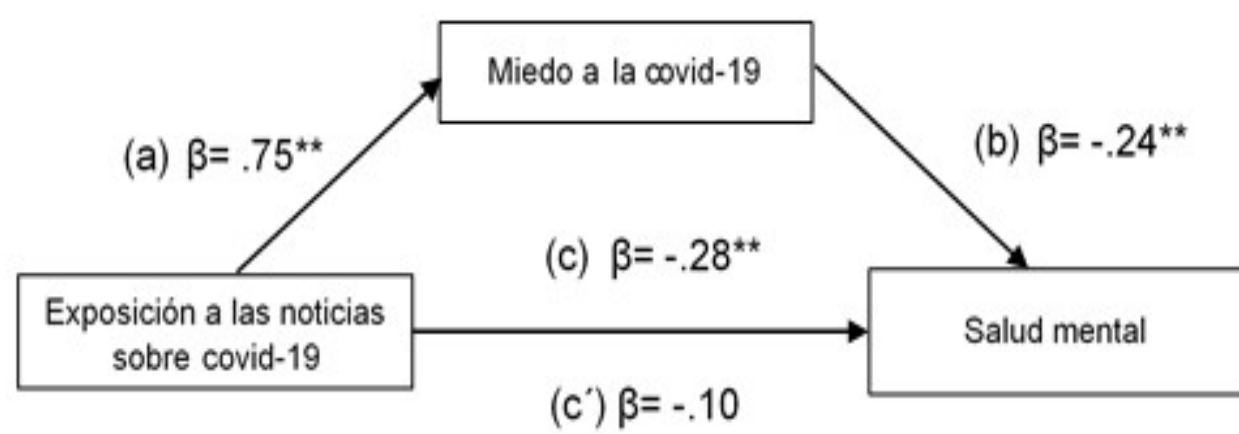

Exposición a las noticias sobre covid- $19 \rightarrow$ Miedo a la covid- $19 \rightarrow$ Salud mental

$$
\begin{gathered}
\text { Efecto indirecto (ab) } \\
B=-.18, S E=.05,95 \% \text { IC }[-.29 ;-.08]
\end{gathered}
$$

\section{Análisis mediacional}

La regresión lineal reveló que el miedo a la covid-19 juega un papel mediador en relación con el número de horas viendo y/o escuchando información de la covid-19 y la salud mental. La cantidad de tiempo viendo y/o escuchando información de la covid-19 (VI) es un buen predictor de la variable mediadora de miedo a la covid-19 ( $\beta$ $\left.=0,75 ; \mathrm{t}=3,77, p<0,001^{* *}\right)$; a su vez, esta tiene un efecto predictor sobre la salud mental $\left(\beta=-0,24 ; t=-13,57, p<0,001^{* *}\right)$. En cuanto al efecto indirecto, se muestra que, en el intervalo de confianza, el cero no se incluye $(B=-0,18$, SE $=0,05 ; 95 \%$ IC $[-0,29$; $-0,08]$ ), por lo que se puede argumentar que el miedo a la covid-19 tiene un papel mediador sobre el número de horas viendo y/o escuchando información de la covid-19 y la salud mental.

Por otro lado, se observó que el número de horas viendo y/o escuchando información de la covid-19 no tuvo un efecto directo positivo con la salud mental $(\beta=-0,10 ; t=-$ $1,184, p=0,23$ ); sin embargo, se considera el efecto total del mediador sobre las variables independiente $y$ dependiente $\left(\beta=-0,24 ; t=-13,57, p<0,001^{* *}\right)$. 


\section{DISCUSIÓN}

La salud mental se ha visto afectada con la pandemia por covid-19 ${ }^{(4,5)}$, situación que amerita el diseño de políticas públicas para garantizar la salud mental como uno de los derechos de todo ser humano. Su atención implica recoger evidencia científica de sus variables predictoras, por ello se investigó el papel mediador del miedo a la covid19 , en la relación entre la exposición a las noticias sobre la covid-19 y la salud mental de la población peruana.

El hallazgo más relevante del estudio permitió comprobar la hipótesis de que el miedo a la covid-19 actúa como mediador entre la exposición a las noticias sobre la covid-19 y la salud mental. Esto indica que observar o escuchar noticias sobre la covid-19 en los medios activa el miedo a la covid-19, a nivel emocional y de expresiones somáticas, lo que a su vez se constituye en un predictor negativo de la salud mental; es decir se afecta el bienestar integral y pueden manifestarse síntomas psicopatológicos. Sin embargo, de acuerdo a un antecedente, el miedo y el conocimiento percibido sobre la covid-19 median la relación entre la exposición a los medios y las conductas preventivas contra la covid-19 (17). Ello indica que el miedo prepara al organismo para su protección ${ }^{(28)}$ mediante conductas preventivas; aunque el afecto negativo constante puede generar complicaciones ${ }^{(21)}$, lo que explicaría el hallazgo reportado.

En el presente estudio, el efecto hallado del miedo como mediador fue total. Por ello, una menor exposición a los medios puede ser favorable, ante un escenario de muertes y sufrimiento en condiciones dramáticas, con una importante carga emocional (21). Además, a este panorama se suman: la presencia de síntomas psicopatológicos, producto del distanciamiento social y confinamiento(4), el propio estado de salud o de la familia o amigos, estar a cargo de familiares contagiados por la covid-19, la incertidumbre de no saber el tiempo de duración que tendrá la pandemia (29) y la crisis económica vivida, como consecuencia de la pandemia, especialmente por ser un país con alta informalidad.

Por tanto, la exposición a noticias generadoras de zozobra y miedo incrementan la victimización indirecta, referida a la posibilidad de ser la próxima víctima del virus (29). De esta manera, el miedo a la covid-19 se relaciona negativamente y con un tamaño de efecto grande con la salud mental. El miedo está vinculado a la incertidumbre y a la percepción del riesgo de contagio de la enfermedad, no exclusivo de personas mayores o con enfermedades previas, así como a la experiencia de haber perdido a un familiar o persona allegada ${ }^{(30)}$. La presencia de esta reacción emocional, que es una de las más vinculadas a la pandemia ${ }^{(6,8)}$, se relaciona directamente con la presencia de problemas de salud mental ${ }^{(23)}$ e inversamente con la calidad de vida ${ }^{(24)}$.

Si bien las investigaciones previas señalan que los medios de comunicación no solo cumplen un rol informativo, sino que son capaces de infundir miedo en las personas expuestas a noticias sobre la covid-19 $(12,19)$, al difundir información imprecisa e infundada (17); en el presente estudio, la relación entre la exposición a noticias sobre la covid-19 y el miedo a la covid-19 fue de tamaño de efecto pequeño. Este hallazgo puede explicarse en función de que el estado emocional ante la exposición a las noticias, depende del contenido que se divulga ${ }^{(25)}$, así como del desarrollo de las 
competencias informacionales, que implican analizar críticamente el contenido de la información, así como la fuente de la que procede ${ }^{(16)}$.

El estudio no encontró evidencia suficiente para la relación entre la exposición a las noticias sobre la covid-19 y la salud mental. Como ya se refirió previamente la relación entre ambas variables está mediada en su totalidad por el miedo a la covid-19. Aun cuando existe evidencia de que quienes comparten información sobre la covid-19 experimentan mayor afecto negativo, ansiedad y estrés ${ }^{(25)}$ y que quienes siguen las noticias sobre la pandemia son más vulnerables a padecer afecciones en su salud mental (13), los efectos varían de acuerdo al contenido de la información divulgada (25).

La difusión de información sobre la gravedad de la enfermedad e informes hospitalarios se asocia a un mayor afecto negativo y a la depresión, mientras que la información brindada por especialistas sobre el conocimiento de la enfermedad y las medidas de prevención se relacionó a menor depresión, a una afectividad positiva (25) y una mejor salud mental ${ }^{(14)}$. Este aspecto es de relevancia para considerarse en el diseño de políticas públicas que permitan enfrentar los problemas de salud mental, especialmente en un país -como es Perú- en el que la poca inversión en salud y la pandemia, han dejado entrever la vulnerabilidad individual y colectiva de su población.

Una de las limitaciones del estudio está referida al diseño explicativo no experimental, que carece del control de todas las variables extrañas que garanticen la validez interna de la investigación. Además, al haber sido transversal no fue posible registrar los cambios en el tiempo, desde que la covid-19 fue un brote y luego una pandemia, en una primera, segunda o inicios de una tercera ola.

Otro aspecto de interés es el momento de la recogida de datos, donde la absoluta mayoría de los participantes no se habían contagiado de la covid-19; sin embargo, sería importante replicar el estudio considerando que el escenario está cambiando, luego de un aumento alarmante de la cantidad de contagiados y fallecidos.

Un aspecto limitante del estudio es que la muestra no ha sido representativa de las tres regiones geográficas del Perú. Si bien es cierto, procedió de 6 departamentos del Perú (de un total de 24), 5 de ellos eran de la costa, 1 de la sierra y ninguno de la selva. Además, el promedio de edades de los participantes era de 30.13 años (DE $=$ 10.31). Por lo que futuros estudios podrían considerar el análisis por grupo etario y hacer el estudio con una muestra representativa de toda la población peruana.

Otra limitación fue no haber analizado la variable sexo, considerando que la mayor parte de los participantes fueron mujeres. El análisis por sexo sería relevante en futuras investigaciones, considerando el antecedente que refiere a las mujeres son las que regulan mejor su miedo, en comparación con los varones ${ }^{(12)}$.

\section{CONCLUSIONES}

A modo de conclusión, conviene resaltar la importancia que cumplen los medios tradicionales e internet en la difusión de la información que no solo contribuye a conocer la enfermedad y a asumir las medidas de prevención de su contagio, sino que repercute en la generación de miedo, que afecta a la salud mental de la población, la misma que se encuentra afectada por las consecuencias del virus en 
ellos mismos o en sus familiares o por las medidas de cuarentena impuestas. Por ello, sería conveniente evitar la infodemia al implementar políticas públicas que articule la participación de las instituciones educativas, científicas y culturales para lograr la alfabetización informacional (16), así como promover la recepción crítica de la información, reconocer las fuentes fidedignas y educar al público en la importancia de evitar difundir información cuestionable en internet que afecte a la comunidad.

\section{REFERENCIAS}

1. Bakioğlu F, Korkma, O, Ercan H. Fear of COVID-19 and Positivity: Mediating Role of Intolerance of Uncertainty, Depression, Anxiety, and Stress. Int J Ment Health Addict. 2020. DOI: https://doi.org/10.1007/s11469-020-00331-y

2. Falcón- Hernández $A$, Navarro-Machado $V$, Díaz-Brito $A$, Delgado-Acosta $H$, Valdés-Gómez M. Pesquisa activa masiva poblacional para la COVID-19. Experiencia con estudiantes de las ciencias médicas. Cienfuegos, 2020. MediSur. 2020; 18(3): 381-387. Disponible en: http://scielo.sld.cu/scielo.php?script=sci arttext\&pid=S1727897X2020000300381\&lng=es\&tlng=es.

3._Cao W, Fang Z, Hou G, Han M, Xu X, Dong J. The psychological impact of the COVID-19 epidemic on college students in China. Psychiatry Res. 2020; 287(112934). DOI: https://doi.org/10.1016/j.psychres.2020.112934

4. Cavicchioli M, Ferrucci R, Guidetti M, Canevini MP, Pravettoni G, Galli F. What Will $\mathrm{Be}$ the Impact of the COVID-19 Quarantine on Psychological Distress? Considerations Based on a Systematic Review of Pandemic Outbreaks. Healthcare. 2021; 9, 101. DOI: https://doi.org/10.3390/healthcare9010101

5. Wang C, Pan R, Wan X, Tan Y, Xu L, Ho C, Ho R. Immediate Psychological Responses and Associated Factors during the Initial Stage of the 2019 Coronavirus Disease (COVID-19) Epidemic among the General Population in China. Int J of Environ Res Public Health. 2020; 17(5): 1-25. DOI: https://doi.org/10.3390/ijerph17051729

6. Ramírez-Ortiz J, Castro-Quintero D, Lerma-Córdoba C, Yela-Ceballos F, EscobarCórdoba F. Mental health consequences of the COVID-19 pandemic associated with social isolation. Colombian Journal of Anesthesiology. 2020; 48(4): e930. DOI: https://doi.org/10.5554/22562087.e930

7. Zhang Z, Feng $\mathrm{Y}$, Song $\mathrm{R}$, et al. Prevalence of psychiatric diagnosis and related psychopathological symptoms among patients with COVID-19 during the second wave of the pandemic. Global Health. 2021; 17(44). DOI: https://doi.org/10.1186/s12992-021-00694-4

8. Lu W, Wang H, Lin Y, Li L. Psychological status of medical workforce during the COVID-19 pandemic: A cross-sectional study. Psychiatry Research. 2020; 288(112936). DOI: https://doi.org/10.1016/j.psychres.2020.112936.

9. Dubey S, Biswas P, Ghosh R, Chatterjee S, Dubey M, Chatterjee S, Lahirim D, Lavie C. Psychosocial impact of COVID-19. Diabetes Metab Syndr. 2020; 14(5): 779-788. DOI: https://doi.org/10.1016/j.dsx.2020.05.035

10. Korkmaz H, Güloğlu B. The role of uncertainty tolerance and meaning in life on depression and anxiety throughout Covid-19 pandemic. Pers. Individ. Differ. 2021; 179, 110952. DOI: https://doi.org/10.1016/j.paid.2021.110952

11. Gallegos de San Miguel M, Zalaquett C, Luna-Sánchez S, Mazo-Zea R, OrtizTorres B, Penagos-Corzo J, Portillo N, Torres Fernández I, Urzúa A, Morgan Consoli M, Hayes AF. Introduction to mediation, moderation, and conditional 
process analysis: a regression based approach. New York: Guilford Publications; 2018.

12. Mejía CR, Rodriguez-Alarcon JF, Garay-Rios L, Enriquez-Anco M, Moreno A, Huaytan-Rojas K, Huancahuari-Nañacc N, Julca-Gonzales A, Alvarez CH, ChoqueVargas $J$, Curioso $W H$. Percepción de miedo o exageración que transmiten los medios de comunicación en la población peruana durante la pandemia de la COVID-19. Rev Cuba de Investig Bioméd. 2020; 39(2)e698. Disponible en: http://scielo.sld.cu/pdf/ibi/v39n2/1561-3011-ibi-39-02-e698.pdf

13. Fiorillo A, Gorwood P. The consequences of the COVID-19 pandemic on mental health and implications for clinical practice. European Psychiatry. 2020; 63(1): 32. DOI: http://doi.org/10.1192/j.eurpsy.2020.35

14. Sarabia S. La salud mental en los tiempos del coronavirus. Rev Neuropsiquiatria. 2020; 83(1): 3-4. DOI: https://doi.org/10.20453/rnp.v83i1.3680.

15. Organización Panamericana de la Salud y Organización Mundial de la Salud. Entender la infodemia y la desinformación en la lucha contra el covid-19. 2020. Disponible en: https://iris.paho.org/bitstream/handle/10665.2/52053/FactsheetInfodemic spa.pdf?sequence $=14 \&$ isAllowed $=y$

16. Alfonso-Sánchez I, Fernández-Valdés M. Comportamiento informacional, infodemia y desinformación durante la pandemia de COVID-19. Anales de la Academia de Ciencias de Cuba. 2020; 10(2). Disponible en: http://www.revistaccuba.sld.cu/index.php/revacc/article/view/882

17. Melki J, Tamim H, Hadid D, Farhat S, Makki M, Ghandour L, Eveline H. Media Exposure and Health Behavior during Pandemics: The Mediating Effect of Perceived Knowledge and Fear on Compliance with COVID-19 Prevention $\begin{array}{llll}\text { Measures. Health } 2020 . & \text { Communication. }\end{array}$ http//doi.org/10.1080/10410236.2020.1858564

18. Yang C, Dillard JP, Li R. Understanding Fear of Zika: Personal, Interpersonal, and Media Influences. Risk Analysis. 2018; 38(12): 35-45. DOI: https://doi.org/10.1111/risa.12973

19. Riaño-Vargas $A$, Rocha-Salamanca $P$. La estadística en los medios de comunicación como detonante del miedo en estudiantes universitarios colombianos. Revista Científica. 2020; 1(37): 6-17. DOI: https://doi.org/10.14483/23448350.15519

20. Organización Mundial de la Salud (OMS). Salud mental: fortalecer nuestra respuesta. 30 de marzo de 2018. Disponible en: https://www.who.int/es/newsroom/fact-sheets/detail/mental-health-strengthening-our-response

21. Buitrago Ramírez F, Ciurana Misol R, Fernández Alonso M, Tizón J.L. Pandemia de la COVID-19 y salud mental: reflexiones iniciales desde la atención primaria de salud española. Atención primaria. 2021; 53(1): 89-101. DOI: https://doi.org/10.1016/j.aprim.2020.06.006

22. Chi X, Liang K, Chen S-T, Huang Q, Huang L, Yu Q, Jiao C, Guo T, Stubbs B, Mahbub M, Yeung A, Kong Z, Zou L. Mental health problems among Chinese adolescents during the COVID-19: The importance of nutrition and physical activity. Int J Clin Health Psychol. 2021; 21(3): 100218. DOl: https://doi.org/10.1016/j.ijchp.2020.100218

23. Servidio R, Giuseppina M, Palermiti AL, Costabile A. Fear of COVID-19, depression, anxiety, and their association with Internet addiction disorder in a sample of Italian students. Journal of Affective Disorders Reports. 2021; 4: 100097. DOI: https://doi.org/10.1016/j.jadr.2021.100097 
24. Somayyeh N, Mojgan M. Relationship of fear of COVID-19 and pregnancy-related quality of life during the COVID-19 pandemic. Arch Psychiatr Nurs. 2021; 35(4): 364-368. DOI: https://doi.org/10.1016/j.apnu.2021.05.006

25. Chao M, Xue D, Liu T, Yang H, Hall BJ. Media use and acute psychological outcomes during COVID-19 outbreak in China. J. Anxiety Disord. 2020; 74: 102248. DOI: https://doi.org/10.1016/j.janxdis.2020.102248

26. Lara A, Navarro C, Mondragón L, Rubí N, Lara C. Validez y confiabilidad del MHI5 para evaluar la depresión de mujeres en primer nivel de atención de Salud Mental. Salud Ment. 2002; 25(6): 13-20. Disponible en: https://bit.ly/2BkEIKi

27. Huarcaya-Victoria J, Villarreal-Zegarra D, Podestà, A. et al. Propiedades psicométricas de una versión española de la escala de miedo al COVID-19 en población general de Lima, Perú. Adicción a la salud Int J Ment. 2020. DOI: https://doi.org/10.1007/s11469-020-00354-5

28. Adolphs R, Mlodinow L, Feldman L. What is an emotion? Current Biology. 2019; 19(20): R1060-R1064. DOI: https://doi.org/10.1016/j.cub.2019.09.008

29. Ribot VC, Chang-Paredes N, González-Castillo AL. Efectos de la COVID-19 en la salud mental de la población. Rev Haban Cienc Méd. 2020; 19(Supl.): e3307. Disponible en: http://www.revhabanera.sld.cu/index.php/rhab/article/view/3307

30. Botero-Rodriguez F, Mosquera-Quiñónez M, Martínez Torres LM, Bolívar-Moná S, Jovel G, Vargas L, Franco O, Gómez-Restrepo C. Análisis de percepciones y repercusiones emocionales en usuarios de Twitter en Colombia durante la pandemia de COVID-19. Rev Colomb Psiquiatr. 2021. DOI: https://doi.org/10.1016/j.rcp.2021.05.005 